\title{
Inventorizations of Weed Species from Wheat Crop Fields of District Fatehgarh Sahib, Punjab (India)
}

\author{
Yadvinder Singh $^{1} *$ and Rai Singh ${ }^{2}$ \\ ${ }^{1}$ Department of Botany and Environmental Science, Sri Guru Granth Sahib World University, \\ Fatehgarh Sahib-140406, Punjab, India \\ ${ }^{2}$ Pesently at Department of Botany, Panjab University, Chandigarh -160014, Punjab, India \\ *Corresponding author
}

\section{A B S T R A C T}

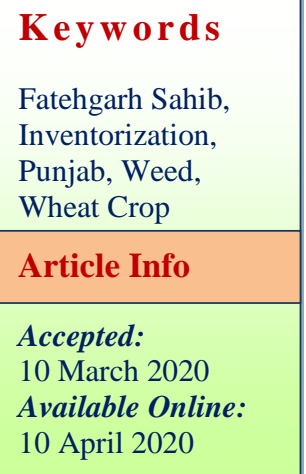

\section{Introduction}

Wheat (Triticum aestivum L.) is an important staple food crop of the world. It offers high content of proteins and also contains carbohydrates in the form of starch (Singh et al., 2015). Sometimes, Wheat crop suffers a severe loss due to heavy weed infestation. Weeds are nuisance in crop fields as they compete with the main crop for light, space and nutrients (Singh and Singh 2019). Weeds also reduce crop yield quality and also interfere in harvesting. The species like Avena sativa, Chenopodium album, Fumaria indica and Phalaris minor etc are common weeds in wheat crop fields (Ahmed and Shaikh, 2003; Khobragad and Sathawane, 2014).

Weeds are those plants with harmful or objectionable habits or characteristics which grow where they are not wanted, usually in places where it is desired that something else should grow (Zimdahl, 2007; Walia, 2009). Weed is a plant which shares the nutrients, space and light of main crop (Singh et al., 2015). Weeds are found inside the crop and 
surroundings also. Weeds like Convolvulus microphyllus root system is coiled to increase surface area and length for increased absorption efficiency. Grasses such as Cyanodon dactylon, Cyperus rotundus are known to survive under very dry conditions. Some weeds have ecotypes with differential resistance to insect attack and varied nutrient absorbing capacities (Zimdahl, 2007). The study of weed plants also provides knowledge about the importance of weed because some weeds have large number of ethnobotanical values (Shah et al., 2016).

It can help us develop new products in pharmaceuticals and food industries (Kendler et al., 1992; Anjalam et al., 2016). Some weeds commonly used by local peoples for food, medicine and fodder tec. For better management of weeds, it is necessary to study their morphology, physiology, systematics, ecology, and ethnobotany (Singh and Singh 2019).

Complete destruction of weeds from the crops by physically, chemically or biologically are dangerous alarming in the loss of biodiversity. Management of weed plants is also important because without management weeds affect the yield and quality of the main crop. So, there is a need of integrated management of weed species without any loss of biodiversity. The main objectives of the present study is to prepare an inventory of weed species growing in wheat crop fields of Fatehgarh Sahib to helps the farmers for designing a suitable management strategies without any harming the plant diversity of the study area.

\section{Materials and Methods}

\section{Study area}

Wheat crop fields of different localities (Mandi Gobindgarh, Amloh, Chunnikalan, Sirhind, Khamanon, Bassi Pathana and
Charnarthal) in District Fatehgarh Sahib, Punjab were surveyed (Fig. 1).

\section{Collection}

The study was conducted during November 2017 to April 2018 to explore weed diversity. Standard methods were followed for collection of weed species. The species were collected depending upon their requirement and availability. Small plants were collected in whole form with root, stem, leaves, flowers and fruits. For collection of large shrubs it is impossible to collect in whole form therefore only twig of plant which includes stem, leaves, flowers and fruits were collected.

\section{Herbarium preparation}

After collection of plant specimens they were pressed using herbarium press. The plant specimens were placed in between three to five layers of papers. The paper sheets were changed at regular interval of 8 days. After proper drying and pressing, the plant specimens were mounted on herbarium sheets for preparation of herbarium. Herbarium sheets were protected against damages from insect and fungal attack by poison treating them with $1 \%$ mercuric chloride solution. Naphthalene balls were also used to protect herbarium from insects.

\section{Identification}

The collected plants species were identified on the basis of available literature like Nair (1978); Meenakshi and Sharma (1985); Sidhu and Bir (1991); Rawat et al., (2013); Kaur et al., (2017). The herbarium specimens of identified plant species were submitted to the Herbarium, Department of Botany and Environmental Science, Sri Guru Granth Sahib World University, Fatehgarh Sahib, Punjab. 


\section{Results and Discussion}

During the survey, 51 weed species were collected from wheat crop fields of different regions of district Fatehgarh Sahib. These weed species belongs to 48 genera of 22 families. Out of 51 Species 42 species were belonging to dicot (Ranunculaceae, Papaveraceae, Fumariaceae, Brassicaceae, Caryophyllaceae, Malvaceae, Geraniaceae, Fabaceae, Asteraceae, Primulaceae, Asclepidaceae, Convolvulaceae, Solanaceae, Scrophulariaceae, Lamiaceae, Chenopodiaceae, Amaranthaceae, Polygonaceae, Euphorbiaceae and Cannabaceae) and 09 species belongs to monocot (Cyperaceae and Poaceae).

Family Poaceae and Asteraceae dominate in the study area with 8 species each followed by family Fabaceae with 5 species, Caryophyllaceae and Polygonaceae with 03 species each, Brassicaceae, Malvaceae, Solanaceae, Chenopodiaceae, Amaranthaceae, Convolvulaceae and Polygonaceae 02 species each.

Whereas family Ranunculaceae, Papaveraceae, Fumariaceae, Geraniaceae, Primulaceae, Asclepidaceae, Convolvulaceae, Scrophulariaceae, Lamiaceae, Euphorbiaceae, Cannabaceae and Cyperaceae were monotypic and represented by only 01 species from study area (Table 1).

Genus Convolvulus, Veronica and Rumex are represented by 02 species each whereas Ranunculus, Argemone, Fumaria, Sisymbrium, Cronopus, Stellaria, Spergula, Silene, Malva, Malvastrum, Oxalis, Medicago, Lathyrus, Vicia, Melilotus, Trifolium, Ageratum, Gnaphalium, Parthenium, Artemisia, Cirsium, Sonchus, Conyza, Launaea, Anagallis, Calotropis, Solanum, Nicotiana, Salvia, Chenopodium, Chenopodiastrum, Achyranthes,
Alternanthera, Polygonum, Euphorbia, Cannabis, Cyperus, Poa, Cyanodon, Lolium, Avena, Dichanthium, Polypogon, Cenchrus and Phalaris were represented by only 01 species each (Table 2 and Image 2-3).

The various studies have been conducted on the weed flora of wheat by different works. These studies determined weed species composition and the level of weed infestation in wheat crop (Salonen et al., 2001).

Kirec and Yarci (1999) recorded 93 weeds occurring in wheat from the agricultural areas of Enez (Edirne), Turkey. Dhole et al., (2013) studied 30 weed species which were related to 15 families and 26 genera from the rabi wheat field in the Marathwada region. Alhasi et al., (2017) studied Weed Flora of the Barley (Hordeum vulgare L.) and Wheat (Triticum aestivum L.) fields in Al-Marj Plain Libya. Kumar and Duggal (2017) reported 54 weed species of 50 genera belonging to 22 families from wheat crop field of Narwana region of Haryana. During present study, Phalaris minor was reported in all localities followed by Rumex dentatus and Fumaria indica. Other dominant species were Cronopus didyma, Chenopodium album, Spergula arvensis etc. Phalaris minor was dominant species of irrigated soil during rabi season in Punjab.

Phalaris minor were more dominant and founds in all the wheat crop fields and may decrease the grain yields of wheat if occurs dense in growth. There is large habitat difference in occurrence between one weed species to another like Veronica anagaliisaquatica and Anagallis arvensis etc. are reported only from the water canals. Large number of weed species founds on the bunds of wheat crop fields like Argemone mexicana, Ageratum conyzoides, Solanum nigrum and Nicotiana plumbinifolia etc. Chenopodium album, chenopodium murale and Cronopus didymus were also founds densely in the crop. 
Some of the weeds reported from the study area having ethnobotanical values which were used for different purposes like food, fodder and for diagnosis of some diseases by native peoples. Melilotus indicus have large number of medicinal properties and therefore this is commonly used by the local peoples to cure some skin diseases. Chenopodium album is commonly used as a vegetable. Cyanodon dactylon, Lolium temulentum and Avena sativa were commonly used as a fodder for animals. Melilotus indicus, Calotropis procera and Rumex dentatus were commonly used by local peoples to cure some diseases.

Table.1 Name of family with number of genera and species identified from study area

\begin{tabular}{|c|l|c|c|}
\hline S. No. & Family & Genera & Species \\
\hline $\mathbf{1}$ & Ranunculaceae & 01 & 01 \\
\hline $\mathbf{2}$ & Papaveraceae & 01 & 01 \\
\hline $\mathbf{3}$ & Fumariaceae & 01 & 01 \\
\hline $\mathbf{4}$ & Brassicaceae & 02 & 02 \\
\hline $\mathbf{5}$ & Caryophyllaceae & 03 & 03 \\
\hline $\mathbf{6}$ & Malvaceae & 02 & 02 \\
\hline $\mathbf{7}$ & Geraniaceae & 01 & 01 \\
\hline $\mathbf{8}$ & Fabaceae & 05 & 05 \\
\hline $\mathbf{9}$ & Asteraceae & 08 & 08 \\
\hline $\mathbf{1 0}$ & Primulaceae & 01 & 01 \\
\hline $\mathbf{1 1}$ & Asclepidaceae & 01 & 01 \\
\hline $\mathbf{1 2}$ & Convolvulaceae & 01 & 02 \\
\hline $\mathbf{1 3}$ & Solanaceae & 02 & 02 \\
\hline $\mathbf{1 4}$ & Scrophulariaceae & 01 & 02 \\
\hline $\mathbf{1 5}$ & Lamiaceae & 01 & 01 \\
\hline $\mathbf{1 6}$ & Chenopodiaceae & 02 & 02 \\
\hline $\mathbf{1 7}$ & Amaranthaceae & 02 & 02 \\
\hline $\mathbf{1 8}$ & Polygonaceae & 02 & 03 \\
\hline $\mathbf{1 9}$ & Euphorbiaceae & 01 & 01 \\
\hline $\mathbf{2 0}$ & Cannabaceae & 01 & 01 \\
\hline $\mathbf{2 1}$ & Cyperaceae & 01 & 01 \\
\hline $\mathbf{2 2}$ & Poaceae & 08 & 08 \\
\hline & Total & 48 & 51 \\
\hline & & & \\
\hline & & & 01 \\
\hline
\end{tabular}

Table.2 Taxonomic position, life form, and habit of weeds identified from study area

\begin{tabular}{|l|l|l|l|l|l|l|l|}
\hline $\begin{array}{l}\text { S. } \\
\text { No. }\end{array}$ & Botanical Name & Family & Local Name & Life Form & Habit & Image & $\begin{array}{l}\text { Voucher } \\
\text { number }\end{array}$ \\
\hline 1. & Ranunculus sceleratus L. & Ranunculaceae & Crowfoot & Annual & Herb & $1 \mathrm{a}$ & WU-132 \\
\hline 2. & Argemone maxicana L. & Papaveraceae & Satyanashi & Annual & Herb & $1 \mathrm{~b}$ & WU-133 \\
\hline 3. & Fumaria indica Pugsl. & Fumariaceae & Gajar ghas & Annual & Herb & $1 \mathrm{c}$ & WU-134 \\
\hline 4. & Sisymbrium irio L. & Brassicaceae & Khubkala & Annual & Herb & 1 d & WU-135 \\
\hline 5. & Cronopus didymum L & & Jungli Hallon & Annual & Herb & 1 e & WU-136 \\
\hline 6. & Stellaria media (L.) Vill. & Caryophyllaceae & Phul booti & Annual & Herb & 1 1f & WU-137 \\
\hline
\end{tabular}




\begin{tabular}{|c|c|c|c|c|c|c|c|}
\hline 7. & Spergula arvensis $\mathrm{L}$. & & Kalri booti & Annual & Herb & $1 \mathrm{~g}$ & WU-138 \\
\hline 8. & Silene conoidea $\mathrm{L}$. & & Takla & Annual & Herb & $1 \mathrm{~h}$ & WU-139 \\
\hline 9. & Malva parviflora $\mathrm{L}$ & \multirow[t]{2}{*}{ Malvaceae } & Mallow & Annual & Herb & $1 \mathrm{i}$ & WU-140 \\
\hline 10. & $\begin{array}{l}\text { Malvastrum coromandelianum } \\
\text { (Linn) Garcke }\end{array}$ & & Kharenti & Annual & Herb & $1 \mathrm{j}$ & WU-141 \\
\hline 11. & Oxalis corniculata Linn. & Geraniaceae & Khatti booti & Perennial & Herb & $1 \mathrm{k}$ & WU-142 \\
\hline 12. & Medicago polymorpha $\mathrm{L}$. & \multirow{5}{*}{ Fabaceae } & Maina & Annual & Herb & 11 & WU-143 \\
\hline 13. & Lathyrus aphaca L. & & Jangli matter & Annual & Herb & $1 \mathrm{~m}$ & WU-144 \\
\hline 14. & Vicia sativa $\mathrm{L}$. & & Bakla & Annual & Herb & 1n & WU-145 \\
\hline 15. & Melilotus indicus L & & Senji & Annual & Herb & 10 & WU-146 \\
\hline 16. & Trifolium fragiferum $\mathrm{L}$ & & Jangli barseem & Annual & Herb & $1 \mathrm{p}$ & WU-147 \\
\hline 17. & Ageratum conyzoides $\mathrm{L}$. & Asteraceae & Neelam & Annual & Herb & $1 \mathrm{q}$ & WU-148 \\
\hline 18. & Gnaphalium polycephalum L & & - & Annual & Herb & $1 \mathrm{r}$ & WU-149 \\
\hline 19. & Parthenium hysterophorus L. & & Congress grass & Perennial & $\begin{array}{l}\text { Herb to } \\
\text { shrub }\end{array}$ & $1 \mathrm{~s}$ & WU-150 \\
\hline 20. & $\begin{array}{l}\text { Artemisia scoparia Waldst \& } \\
\text { Kit }\end{array}$ & & Gazer booti & Perennial & $\begin{array}{l}\text { Herb to } \\
\text { shrub }\end{array}$ & $1 \mathrm{t}$ & WU-151 \\
\hline 21. & Cirsium arvense (L.) Scop & & Leh & Annual & $\begin{array}{l}\text { Herb to } \\
\text { shrub }\end{array}$ & $1 \mathrm{u}$ & WU-152 \\
\hline 22. & Sonchus arvensis L. & & - & Annual & Herb & $1 v$ & WU-153 \\
\hline 23. & $\begin{array}{l}\text { Conyza bonariensis (L.) } \\
\text { Cronquist }\end{array}$ & & - & Annual & Herb & $1 \mathrm{w}$ & WU-154 \\
\hline 24. & Launaea nudicaulis Hook. f. & & Hundwaya & Perennial & Herb & $1 \mathrm{x}$ & WU-155 \\
\hline 25. & Anagallis arvensis $L$. & Primulaceae & Billi booti & Annual & Herb & $1 y$ & WU-156 \\
\hline 26. & $\begin{array}{l}\text { Calotropis procera (Willed) } \\
\text { R.Br. }\end{array}$ & Asclepidaceae & Ak & Perennial & Shrub & $2 \mathrm{a}$ & WU-157 \\
\hline 27. & Convolvulus arvensis $\mathrm{L}$. & \multirow[t]{2}{*}{ Convolvulaceae } & Hirankhuri & Annual & Herb & $2 b$ & WU-158 \\
\hline 28. & Convolvulus pluricaulis Choisy & & Shunkhpushpi & Annual & Herb & $2 \mathrm{c}$ & WU-159 \\
\hline 29. & Solanum nigrum $\mathrm{L}$. & \multirow[t]{2}{*}{ Solanaceae } & Makoi & Perennial & $\begin{array}{l}\text { Herb to } \\
\text { Shrub }\end{array}$ & $2 \mathrm{~d}$ & WU-160 \\
\hline 30. & Nicotiana plumbaginifolia Viv. & & $\begin{array}{l}\text { Jungli } \\
\text { tambacco }\end{array}$ & Annual & Herb & $2 \mathrm{e}$ & WU-161 \\
\hline 31. & Veronica anagallis-aquatica L. & \multirow[t]{2}{*}{ Scrophulariaceae } & - & Annual & Herb & $2 \mathrm{f}$ & WU-162 \\
\hline 32. & Veronica agrestis L. & & - & Annual & Herb & $2 g$ & WU-163 \\
\hline 33. & Salvia plebeia $\mathrm{R}$. Br. & Lamiaceae & - & Annual & Herb & $2 \mathrm{~h}$ & WU-164 \\
\hline 34. & Chenopodium album $\mathrm{L}$. & \multirow[t]{2}{*}{ Chenopodiaceae } & Bathu & Annual & Herb & $2 \mathrm{i}$ & WU-165 \\
\hline 35. & Chenopodiastrum murale L. & & Khartua & Annual & Herb & $2 \mathrm{j}$ & WU-166 \\
\hline 36. & Achyranthes aspera $\mathrm{L}$. & \multirow[t]{2}{*}{ Amaranthaceae } & Puthkanda & Annual & Herb & $2 \mathrm{k}$ & WU-167 \\
\hline 37. & $\begin{array}{l}\text { Alternanthera philoxeroides } \\
\text { Griseb }\end{array}$ & & - & Annual & Herb & 21 & WU-168 \\
\hline 38. & Polygonum plebeium R. Br. & \multirow[t]{3}{*}{ Polygonaceae } & Gulabi booti & Annual & Herb & $2 \mathrm{~m}$ & WU-169 \\
\hline 39. & Rumex dentatus L. & & Jungli palak & Annual & Herb & $2 n$ & WU-170 \\
\hline 40. & Rumex spinosus $\mathrm{L}$ & & Kandiali & Annual & Herb & 20 & WU-171 \\
\hline 41. & Euphorbia helioscopia L & Euphorbiaceae & Kour Gandhal & Annual & Herb & $2 p$ & WU-172 \\
\hline 42. & Cannabis sativa $\mathrm{L}$. & Cannabinaceae & Bhung & Perennial & $\begin{array}{c}\text { Herb to } \\
\text { shrub }\end{array}$ & $2 q$ & WU-173 \\
\hline 43. & Cyperus rotundus $\mathrm{L}$ & Cyperaceae & Murk & Perennial & Herb & $2 \mathrm{r}$ & WU-174 \\
\hline
\end{tabular}




\begin{tabular}{|c|c|c|c|c|c|c|c|}
\hline 44. & Poaannua L & \multirow[t]{8}{*}{ Poaceae } & Bueen & Annual & Herb & $2 \mathrm{~s}$ & WU-175 \\
\hline 45. & Cyanodon dactylon (L.) Pers & & Dhub Ghass & Perennial & Herb & $2 t$ & WU-176 \\
\hline 46. & Lolium temulentum $\mathrm{L}$. & & Rye Ghass & Annual & Herb & $2 u$ & WU-177 \\
\hline 47. & Avena sativa $\mathrm{L}$. & & Jungli jai & Annual & Herb & $2 v$ & WU-178 \\
\hline 48. & $\begin{array}{l}\text { Dichanthium annulatum } \\
\text { (Forssk.) Stapf }\end{array}$ & & - & Perennial & Herb & $2 \mathrm{w}$ & WU-179 \\
\hline 49. & $\begin{array}{l}\text { Polypogon monspeliensis (L.) } \\
\text { Desf }\end{array}$ & & Loomber ghas & Annual & Herb & $2 \mathrm{x}$ & WU-180 \\
\hline 50. & Cenchrus ciliaris .L & & Kutta ghas & Annual & Herb & $2 y$ & WU-181 \\
\hline 51. & Phalaris minor Retz. & & Gulli danda & Annual & Herb & $2 z$ & WU-182 \\
\hline
\end{tabular}

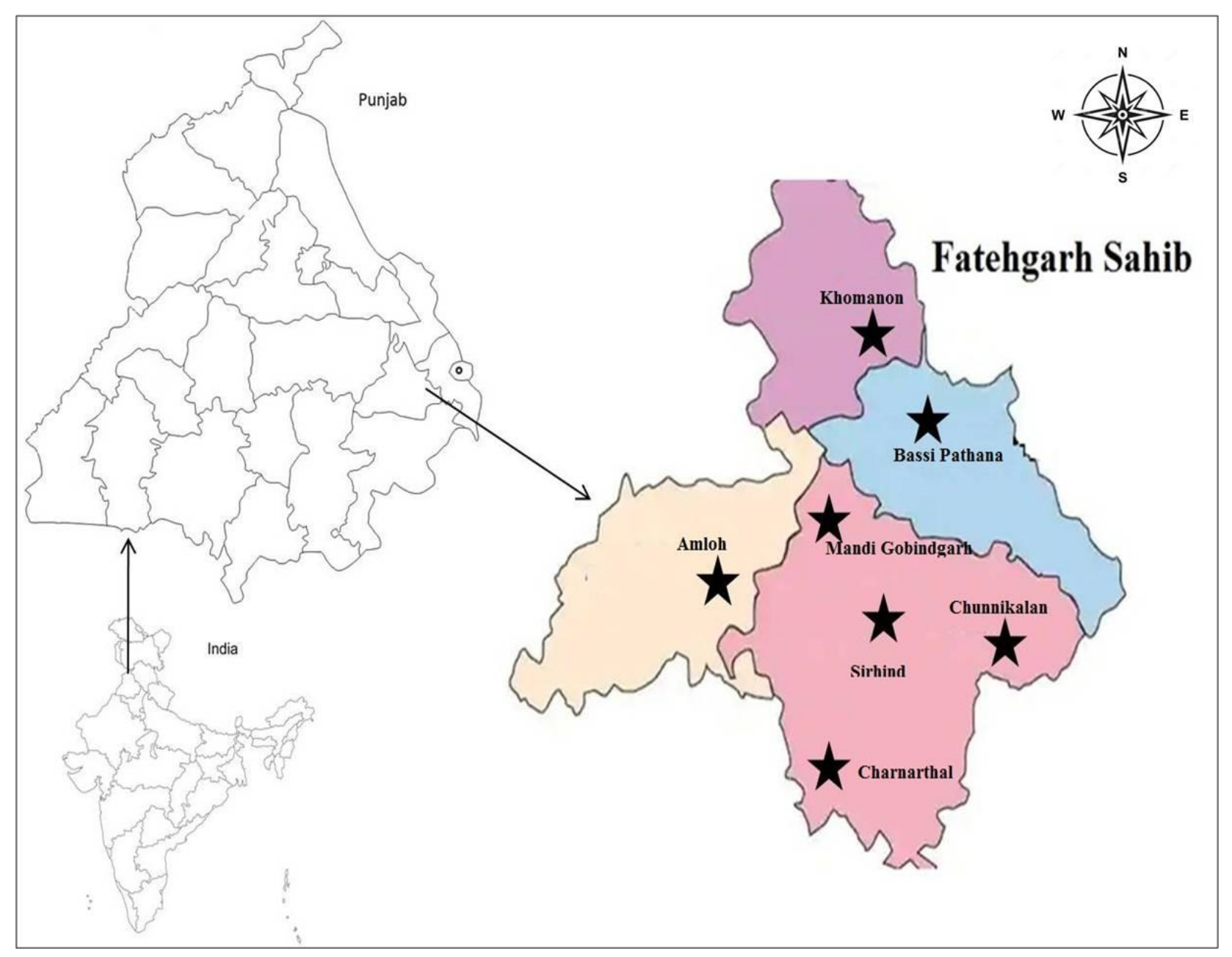

Fig.1 Plant collection sites of district Fatehgarh Sahib, Punjab, India. (Source: www.google.com) 

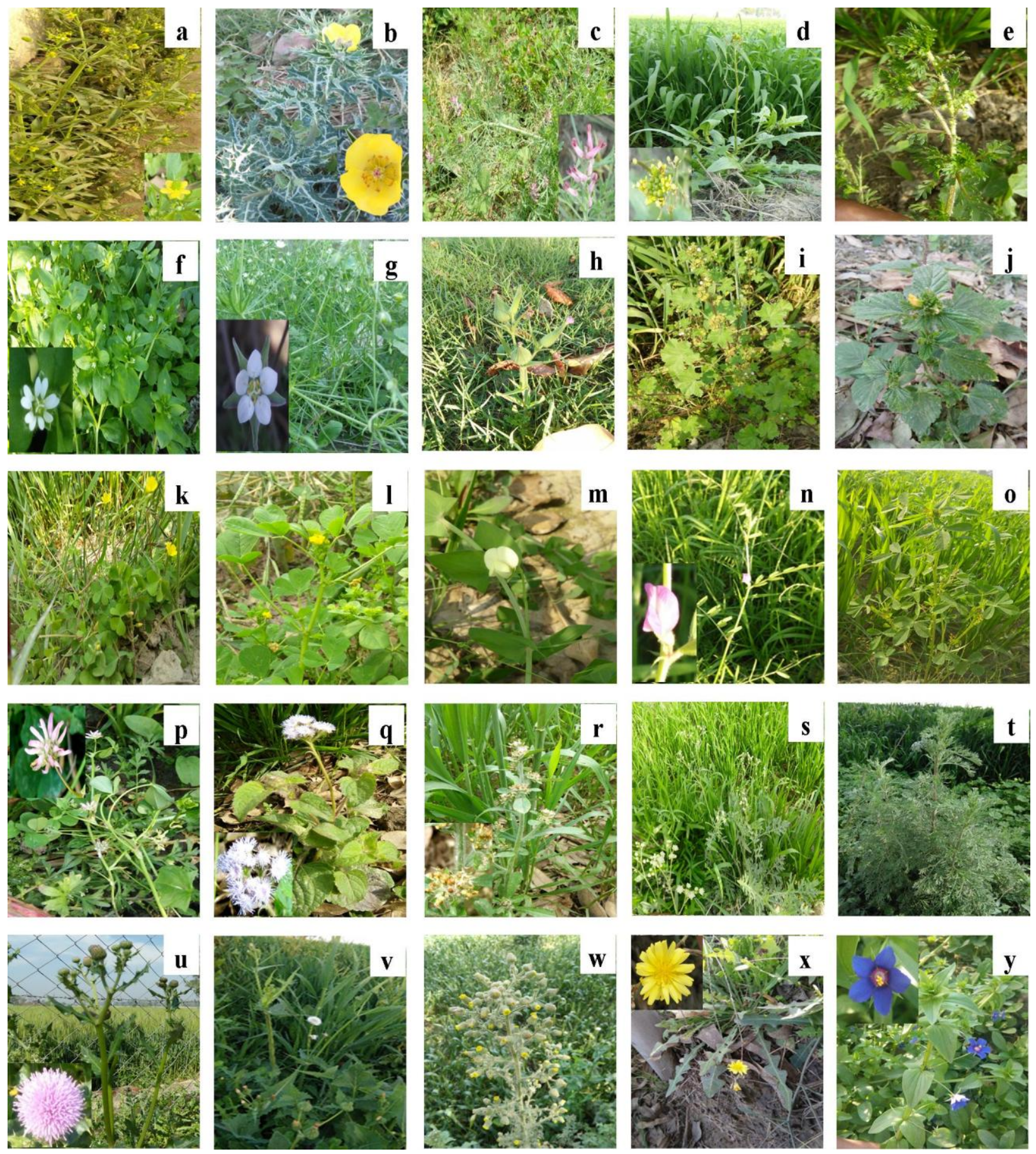

Image.1 Weed plants of wheat crop fields in Fatehgarh Sahib District, Punjab, India:

a-Ranunculus sceleratus | b- Argemone maxicana |c- Fumaria indica |d- Sisymbrium irio | eCronopus didymum | f- Stellaria media | g- Spergula arvensis | h- Silene conoidea | i- Malva parviflora |j-Malvastrum coromandelianum | k- Oxalis corniculata |1-Medicago polymorpha | m- Lathyrus aphaca | n- Vicia sativa | o- Melilotus indicus | p- Trifolium fragiferum | qAgeratum conyzoides | r- Gnaphalium polycephalum | s- Parthenium hysterophorus | tArtemisia scoparia | u- Cirsium arvense | v- Sonchus arvensis | w-Conyza bonariensis | xLaunaea nudicaulis | y- Anagallis arvensis 

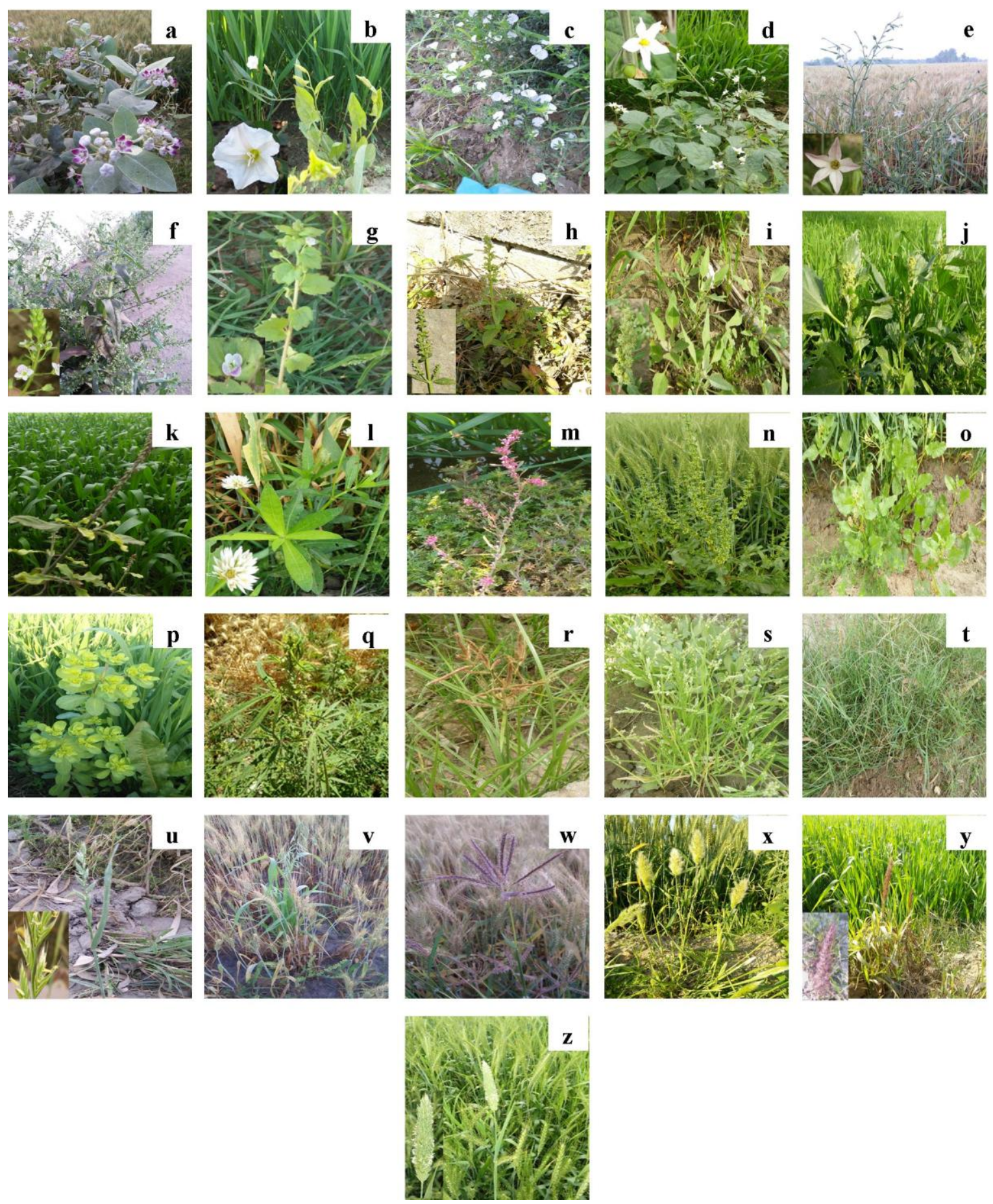

Image.2 Weed plants of wheat crop fields in Fatehgarh Sahib District, Punjab, India: $a$ Calotropis procera $\mid$ b-Convolvulus arvensis $\mid c$-Convolvulus pluricaulis $\mid d$-Solanum nigrum $\mid$ $e$-Nicotiana plumbaginifolia $\mid f$-Veronica anagallis-aquatica $\mid g$-Veronica agrestis $\mid h$-Salvia plebeia $\mid i$ - Chenopodium album $\mid j$-Chenopodiastrum murale $\mid k$-Achyranthes aspera $\mid l$ Alternanthera philoxeroides $\mid m$ - Polygonum plebeium $\mid n$-Rumex dentatus $\mid$ o- Rumex spinosus $\mid$ p- Euphorbia helioscopia $\mid q$ - Cannabis sativa $\mid r$-Cyperus rotundus $\mid s$ - Poa annua $\mid t$ Cyanodon dactylon $\mid u$ - Lolium temulentum $\mid v$-Avena sativa $\mid w$-Dichanthium annulatum $\mid x$ Polypogon monspeliensis | y-Cenchrus ciliaris $\mid z$-Phalaris minor 
The present study was conducted as a first ever attempt from the study area to explore and identify the weeds from wheat crop fields. The present work gives the idea about the total weed diversity of the wheat crop fields of District Fatehgarh Sahib, Punjab. The results obtained from this study clearly established the fact that the diversity of weeds was high and significant. This study will help the farmers and agriculturists of the study area to identify the weeds and thus help in planning a suitable strategy for their control and sustainable management.

\section{Acknowledgements}

Authors wish to express their sincere thanks to the Vice-Chancellor, Sri Guru Granth Sahib World University, Fatehgarh Sahib for providing necessary facilities for the this work.

\section{References}

Ahmed, R. and Shaikh, A.S. 2003. Common weeds of wheat and their control. Pakistan Journal of Water Resources. 7: 73-76.

Alhasi, S.M., El-Barasi, Y.M., Rahil, R.O. and Barrani, M.W. 2017. Weed Flora of the Barley (Hordeum vulgare L.) and Wheat (Triticum aestivum L.) fields in Al-Marj Plain, Libya, ContROL, 1: 0005. DOI: https://dx.doi.org/10.28915/control.000 5.1

Anjalam, A., Kalpana, S., Vijai, D. and Premalatha, S. 2016. Documentation of medicinal plants used by malayali tribes in Kolli hills. International Journal of Advanced Research in Biology Sciences. 3(3): 101-107.

Dhole, J.A., Lone, K.D., Dhole, N.A. and Bodke, S.S. 2013. Studies on weed diversity of Wheat (Triticum aestivum L.) crop fields of Marathwada Region,
International Journal of Current Microbiology and Applied Sciences. 2(6): 293-298.

Kaur, K., Sidhu, M.C. and Ahluwalia, A.S. 2017. Angiospermic diversity in Doaba region of Punjab, India. Journal of Threatened Taxa. 9(8): 10551-10564.

Kendler, B.S., Koritz, H.G. and Gibaldi, A. 1992. Introducing students to ethnobotany. The American Biology Teacher 54(1): 46-50.

Khobragad, D.P. and Sathawane, K.N. 2014. Weed diversity in rabi wheat crop of bhandara District (M.S.) India. International Journal of Life Sciences. Spl. Issues A2: 128-131.

Kirec, M. and Yarci, C. 1999. The flora of the agricultural areas in Enez (Enirne) and environs. Turkish Journal of Botany. 23(1): 53- 62.

Kumar, S. and Duggal, S. 2017. A survey of weed of wheat crops fields in Narwana region, India. International Journal of Scientific Research and Management. 5(8): 6664-6669.

Meenakshi and Sharma, M. 1985. Flora of Ropar District. Dev Publishers, Patiala, Punjab, $267 \mathrm{P}$.

Nair, N.C. 1978. Flora of Punjab Plains. Botanical Survey of India, Howrah. 326 p.

Rawat, L, Manhas, R.K., Kholiya, D. and Kamboj, S.K. 2013. Floristic diversity of Kandi region of Hoshiarpur, Punjab, India. Applied Ecology and Environmental Sciences. 1(4): 49-54.

Salonen, J., Hyvonen, T. and Jalli, H. 2001. Weed flora in organically grown spring cereals in Finland. Agricultural and Food Science. 10 (3): 231-242.

Shah, A.A., Khan, Z., Ramzan, M. and Saba, R. 2016. Ethnoecology of Miani Sahib Graveyard, Lahore City, Punjab, Pakistan. Journal of Bio-resource Management. 3(2): 33-44.

Sidhu, M. and Bir, S.S. 1993. Karyological 
studies on weeds on cultivable lands in Punjab, India. Tropical Plant Science Research. 1: 1-13.

Singh, Y. and Singh, R. 2019. Weed diversity in Rice crop fields of Fatehgarh Sahib District, Punjab, India. Journal of Threatened Taxa. 11(5): 13611-13616.

Singh, C., Singh, P. and Singh, R. 2015.
Mordern Techniques of Raising Field Crops, Oxford \& IBH Publishing Co. Pvt. Ltd., New Delhi. 596 p.

Walia, U.S. 2018. Weed Management. $5^{\text {th }}$ ed. Kalyani Publishers, $425 \mathrm{p}$.

Zimdahl, R.L. 2007. Fundamentals of Weed Science. Academic Press is an imprint of Elsevier, $665 \mathrm{p}$.

\section{How to cite this article:}

Yadvinder Singh and Rai Singh. 2020. Inventorizations of Weed Species from Wheat Crop Fields of District Fatehgarh Sahib, Punjab (India). Int.J.Curr.Microbiol.App.Sci. 9(04): 12451254. doi: https://doi.org/10.20546/ijcmas.2020.904.147 